\title{
GRAVITATIONAL SHEAR, FLEXION, AND STRONG LENSING IN ABELL 1689
}

\author{
Adrienne Leonard, ${ }^{1,2}$ David M. Goldberg, ${ }^{1} \mathrm{~J}_{\text {Ason L }}$ L. HaAga, ${ }^{1}$ and Richard Massey ${ }^{3}$ \\ Received 2007 February 8; accepted 2007 May 25
}

\begin{abstract}
We present a gravitational lensing analysis of the galaxy cluster Abell 1689, incorporating measurements of the weak shear, flexion, and strong lensing induced in background galaxies. This is the first time that a shapelet technique has been used to reconstruct the distribution of mass in this cluster and the first time that a flexion signal has been measured using cluster members as lenses. From weak shear measurements alone, we generate a nonparametric mass reconstruction, which shows significant substructure corresponding to groups of galaxies within the cluster. In addition, our galaxy-galaxy flexion signal demonstrates that the cluster galaxies can be well fit by a singular isothermal sphere model with a characteristic velocity dispersion of $\sigma_{v}=(295 \pm 40) \mathrm{km} \mathrm{s}^{-1}$. We identify a major, distinct dark matter clump, offset by $40 \mathrm{~h}^{-1} \mathrm{kpc}$ from the central cluster members, which was not apparent from shear measurements alone. This secondary clump is present in a parametric mass reconstruction using flexion data alone, and its existence is suggested in a nonparametric reconstruction of the cluster using a combination of strong and weak lensing. As found in previous studies, the mass profile obtained by combining weak and strong lensing data shows a much steeper profile than that obtained from only weak lensing data.
\end{abstract}

Subject headings: cosmology: observations — galaxies: clusters: general — galaxies: photometry — gravitational lensing

\section{INTRODUCTION}

Gravitational lensing studies provide a powerful tool for mapping out the surface mass density of galaxies and clusters of galaxies (e.g., Bradac et al. 2005a, 2005b, 2006; Natarajan \& Springel 2004; Diego et al. 2005a; Cacciato et al. 2006; Abdelsalam et al. 1998). In particular, cluster lensing studies provide constraints on both structure formation models and the mean mass density of the universe. In addition, accurate knowledge of the mass distribution in clusters enables better determination of the relationship between dark matter, gas, and galaxies within the cluster and allows us to probe how well light traces mass. For example, there has been tremendous excitement recently about maps from gravitational lensing of the distribution of baryonic mass and dark matter in large-scale structure (Massey et al. 2007b) and, particularly, the cluster 1E0657-56 (the "Bullet Cluster"; Bradac et al. 2006), in which there is a marked offset between the peaks of baryonic and nonbaryonic mass.

Traditional approaches to weak gravitational lensing focus on estimating the mass of the lens by measuring the induced ellipticity (shear) in images of background galaxies (see, e.g., Kaiser et al. [1995], for a review of the canonical approach inverting ellipticities; Bartelmann \& Schneider [2001] provide an excellent review of the subject). Underlying these approaches is the assumption that any variations in the lensing field over the scale of the galaxy image can be neglected. Flexion has recently been introduced as a means to describe higher order lensing effects which arise as a result of small-scale variations in the lensing field (Goldberg \& Bacon 2005, hereafter GB05; Bacon et al. 2006, hereafter BGRT06; Goldberg \& Leonard 2007, hereafter GL07). The flexion technique enables us to probe structure on smaller scales than does a shear analysis and is particularly useful in galaxy-galaxy lensing studies.

\footnotetext{
${ }^{1}$ Department of Physics, Drexel University, Philadelphia, PA 19104.

2 Institute of Astronomy, University of Cambridge, Cambridge, CB3 OHA, UK.

${ }^{3}$ California Institute of Technology, Pasadena, CA 91125.
}

In this paper we present several mass reconstructions of Abell 1689 , a rich cluster of galaxies at a redshift of $\sim 0.18$, using images taken by the Hubble Space Telescope (HST) Advanced Camera for Surveys (ACS). This cluster has been well studied in the context of both weak and strong lensing, and various parametric models for the mass distribution have been tested (see, e.g., Bardeau et al. 2005; Halkola et al. 2006; Saha et al. 2006; Zekser et al. 2006; Umetsu et al. 2005; Broadhurst et al. 2005a; Diego et al. 2005b; Sharon et al. 2005; King et al. 2002a, 2002b; Dahle et al. 2001; Dye et al. 2001; Taylor \& Dye 1998; Tyson \& Fischer 1995). Recently, Halkola et al. (2007) have investigated the size of individual galaxy halos within the cluster using strong lensing, which is typically difficult to do in clusters using traditional weak lensing techniques.

Likewise, a number of researchers, including the original observing team (Broadhurst et al. 2005b; see also Diego et al. 2005b) have produced parametric and nonparametric mass estimates using strong lensing from the same ACS images in our sample. Most recently, Limousin et al. (2006, hereafter L06) have used these images, as well as images from the CFH12k camera, to model the cluster using both strong and weak lensing measurements and find good agreement in the mass estimates derived from these two sets of measurements.

We present here a nonparametric mass reconstruction of this cluster from shear measurements, as well as a galaxy-galaxy lensing study from measurements of flexion. It is typically very difficult to extract a weak shear galaxy-galaxy signal in clusters of galaxies, as the smooth component of the cluster field dominates. However, flexion is better suited to detect perturbations to this lensing field on smaller scales and, thus, is particularly adept at picking out lensing signals from individual galaxies in the cluster.

We show that our flexion data can be used to construct a parametric mass reconstruction that successfully identifies substructure within the cluster. In addition, we combine our nonparametric shear mass reconstruction with a nonparametric strong lensing reconstruction. The combined convergence map shows significant substructure and suggests the presence of the secondary dark 
matter clump described by L06. In addition, this convergence map shows a steeper radial profile in the center of the cluster than the one generated by shear measurements alone.

This paper is structured as follows. We begin in $\S 2$ with a brief review of flexion formalism and a discussion of our measurement techniques, including an overview of the formalism underlying the shapelets and higher order lensing image's characteristics (HOLICs) methods. We describe our data and processing pipeline in detail in $\S 3$ and present the results of our study in $\S 4$.

\section{MEASURING SHEAR AND FLEXION}

\subsection{Flexion Formalism}

It is helpful to begin by specifying our notation. Consider an image (at $z=\infty$ ) which, in the absence of lensing, would be observed at angular position $\boldsymbol{\beta}$. This can be related to the observed coordinate $\boldsymbol{\theta}$ via the second-order transformation

$$
\beta_{i} \simeq A_{i j} \theta_{j}+\frac{1}{2} D_{i j k} \theta_{j} \theta_{k}
$$

where

$$
\begin{gathered}
\mathbf{A}(\boldsymbol{\theta}) \equiv \frac{\partial \boldsymbol{\beta}}{\partial \boldsymbol{\theta}}=\left(\begin{array}{cc}
1-\kappa-\gamma_{1} & -\gamma_{2} \\
-\gamma_{2} & 1-\kappa+\gamma_{1}
\end{array}\right), \\
D_{i j k}=\partial_{k} A_{i j} .
\end{gathered}
$$

The D operators can be expressed as

$$
\begin{aligned}
D_{i j 1} & =\left(\begin{array}{cc}
-2 \partial_{1} \gamma_{1}-\partial_{2} \gamma_{2} & -\partial_{1} \gamma_{2} \\
-\partial_{1} \gamma_{2} & -\partial_{2} \gamma_{2}
\end{array}\right), \\
D_{i j 2} & =\left(\begin{array}{cc}
-\partial_{1} \gamma_{2} & -\partial_{2} \gamma_{2} \\
-\partial_{2} \gamma_{2} & 2 \partial_{2} \gamma_{1}-\partial_{1} \gamma_{2}
\end{array}\right) .
\end{aligned}
$$

These operators give rise to an asymmetrical distortion in the image, namely, a skewness and a bending or arciness, as well as a shift in the centroid of the light distribution. BGRT06 define two "flexions" as

$$
\begin{gathered}
\mathcal{F}=\partial^{*} \gamma=\left(\partial_{1} \gamma_{1}+\partial_{2} \gamma_{2}\right)+i\left(\partial_{1} \gamma_{2}-\partial_{2} \gamma_{1}\right), \\
\mathcal{G}=\partial \gamma=\left(\partial_{1} \gamma_{1}-\partial_{2} \gamma_{2}\right)+i\left(\partial_{1} \gamma_{2}+\partial_{2} \gamma_{1}\right),
\end{gathered}
$$

where $\partial=\partial_{1}+i \partial_{2}$ is the complex gradient operator defined in BGRT06. The first flexion $\mathcal{F}$ has an $m=1$ rotational symmetry (i.e., it is a vector) and gives rise to a skewness in the light distribution of a galaxy image, as well as a shift in its centroid. It is also simply related to the convergence $\kappa$ by $\mathcal{F}=\partial \kappa$. The second flexion $\mathcal{G}$ has an $m=3$ rotational symmetry and gives rise to bending in the lensed image.

\subsection{Measurement Techniques}

Two distinct methods have been proposed for measuring flexion in real images. The method described in GB05 and BGRT06 involves the decomposition of galaxy images into shapelets (Refregier 2003), followed by an "active" perturbation of coefficients (adopting the terminology of the Shear Testing Program; Heymans et al. 2006; Massey et al. 2007c). A "passive" method, involving high-order moments of a galaxy's shape, referred to as higher order lensing image's characteristics (HOLICs), was first related to flexion by Okura et al. (2007). These techniques have been refined, compared, and discussed in detail in GL07. We also note that others (Irwin \& Shmakova 2005, 2006) have proposed a statistic similar to flexion, using a $\chi^{2}$ minimization technique to fit a polynomial radial profile with first- and second-order perturbations.

\subsubsection{Shapelets}

Several researchers (Refregier 2003; Bernstein \& Jarvis 2002) have proposed techniques for decomposing images into "shapelets," simple basis functions composed of the two-dimensional, Gaussian-weighted Hermite polynomials $B_{n m}(\boldsymbol{\theta})$. Any isolated image $f(\boldsymbol{\theta})$ can be expressed as a sum

$$
f(\boldsymbol{\theta})=\sum_{m n} f_{n m} B_{n m}(\boldsymbol{\theta}),
$$

where the weights $f_{n m}$ are known as "shapelet coefficients." As shown in Refregier (2003), Refregier \& Bacon (2003), Massey \& Refregier (2005), and Massey et al. (2007a), shapelets are an especially useful basis for our purposes, because image transformation operations induced by gravitational lensing typically produce very compact transfers of power between shapelet coefficients. In the shapelets framework, the lensing operators can be expressed elegantly in terms of the quantum mechanical raising and lowering operators, $a$ and $a^{\dagger}$, which are combinations of the $\theta_{i}$ and $\partial_{i}$ operators.

We can express the lens equation as

$$
f=\left(1+\kappa \hat{K}+\gamma_{j} \hat{S}_{j}+\gamma_{i, j} \hat{S}_{i j}^{(2)}\right) f^{\prime},
$$

where $f^{\prime}$ represents the unlensed image, $f$ refers to the lensed image, and the various operators $\hat{K}, \hat{S}_{j}$, and $\hat{S}_{i j}^{(2)}$ are expressed in terms of $a$ and $a^{\dagger}$ (the reader is referred to GB05 for the details).

An important feature of the operators in equation (8) is that the $\hat{K}$ and $\hat{S}_{j}$ operators transfer power in shapelet space such that $|\Delta n|+|\Delta m|=2$; whereas, the second-order operators $\hat{S}_{i j}^{(2)}$ yield $|\Delta n|+|\Delta m|=1$ or 3 . This provides a straightforward mechanism for extracting the first- and second-order signals.

As with a shear analysis, our flexion analysis makes the assumption that any intrinsic flexion signal will be randomly oriented and, thus, will average to zero. This means that we expect that on average the $(n+m=)$ odd shapelet modes will have a zero signal.

In practice, the shapelet series will need to be truncated, in which case there are two parameters that must be specified for the decomposition. These parameters are $n_{\max }$, the maximum order of the shapelet series, and $\beta$, the characteristic scale of the shapelets,

$$
\mathcal{B}_{n m}(\boldsymbol{\theta}) \propto \exp \left(-\frac{\theta_{1}^{2}+\theta_{2}^{2}}{2 \beta^{2}}\right) .
$$

As discussed in Refregier (2003), the optimal choices for $\beta$ and $n_{\max }$ are

$$
\begin{gathered}
\beta \simeq \sqrt{\theta_{\max } \theta_{\min }}, \\
n_{\max } \simeq \frac{\theta_{\max }}{\theta_{\min }}-1,
\end{gathered}
$$

where $\theta_{\max }$ and $\theta_{\min }$ are the maximum and minimum scales on which one expects to resolve structure in the image, respectively. For the data used here, we find $\theta_{\min }=1.0$ pixels and $\theta_{\max }=$ $1.5\left(a^{2}+b^{2}\right)^{1 / 2}$ give good shapelet reconstructions of our galaxy 
images, where $a$ and $b$ are the semimajor and semiminor axes of the object as measured during source extraction, respectively.

Images also need to be corrected for point spread function (PSF) and pixelization effects, which both tend to dilute the lensing signal. One of the advantages of the shapelets technique is that an explicit PSF deconvolution, and an integration within pixels, can be carried out prior to any parameter estimation. Refregier \& Bacon (2003) give an explicit form for the deconvolution, and Massey \& Refregier (2005) derive the form of the integrals. We perform these tasks using the publicly available IDL software from the shapelets Web site. ${ }^{4}$

\subsubsection{HOLICS}

We have discussed above how shapelets can be used to measure both the shear and the flexion of a lensed image. Historically, however, shear has primarily been measured using combinations of weighted moments of the image. Likewise, the HOLICs technique proposed by Okura et al. (2007) and subsequently refined in GL07 provides a straightforward and fast way of estimating flexion using weighted moments of the light distribution of galaxies. Okura et al. (2007) define the complex terms

$$
\begin{gathered}
\zeta \equiv \frac{\left(Q_{111}+Q_{122}\right)+i\left(Q_{112}+Q_{222}\right)}{\xi}, \\
\delta \equiv \frac{\left(Q_{111}-Q_{122}\right)+i\left(3 Q_{112}-Q_{222}\right)}{\xi},
\end{gathered}
$$

where

$$
\xi \equiv Q_{1111}+2 Q_{1122}+Q_{2222}
$$

As pointed out in GL07, the relationship between the above quantities and flexion estimators is best expressed as a matrix equation,

$$
\mathcal{M}\left(\begin{array}{c}
\mathcal{F}_{1} \\
\mathcal{F}_{2} \\
\mathcal{G}_{1} \\
\mathcal{G}_{2}
\end{array}\right)=\left(\begin{array}{l}
\zeta_{1} \\
\zeta_{2} \\
\delta_{1} \\
\delta_{2}
\end{array}\right),
$$

where $\mathcal{M}$ is a $4 \times 4$ matrix whose elements are proportional to linear combinations of $Q_{i j k l}$ and $Q_{i j} Q_{k l}$. The reader is referred to GL07 for the explicit forms of these elements.

Equation (14) describes how flexion could be estimated from unweighted moments in the absence of any measurement noise. However, when dealing with real images, and particularly those dominated by sky noise, measurement of these moments is inherently noisy. It is thus necessary to include a weighting function in our measurements of the moments, which gives higher weighting to the central region of the postage stamp of a galaxy (where the actual image lies) than to the extreme regions (which we expect to be dominated by noise). We use a Gaussian filter

$$
W(\boldsymbol{\theta})=\frac{1}{2 \pi \sigma_{W}} \exp \left(-\frac{\theta_{1}^{2}+\theta_{2}^{2}}{2 \sigma_{W}^{2}}\right)
$$

and define the weighted moments as

$$
\hat{Q}_{i j}=\frac{1}{\hat{F}} \int\left(\theta_{i}-\overline{\theta_{i}}\right)\left(\theta_{j}-\overline{\theta_{j}}\right) f(\boldsymbol{\theta}) W(\boldsymbol{\theta}) d^{2} \boldsymbol{\theta} .
$$

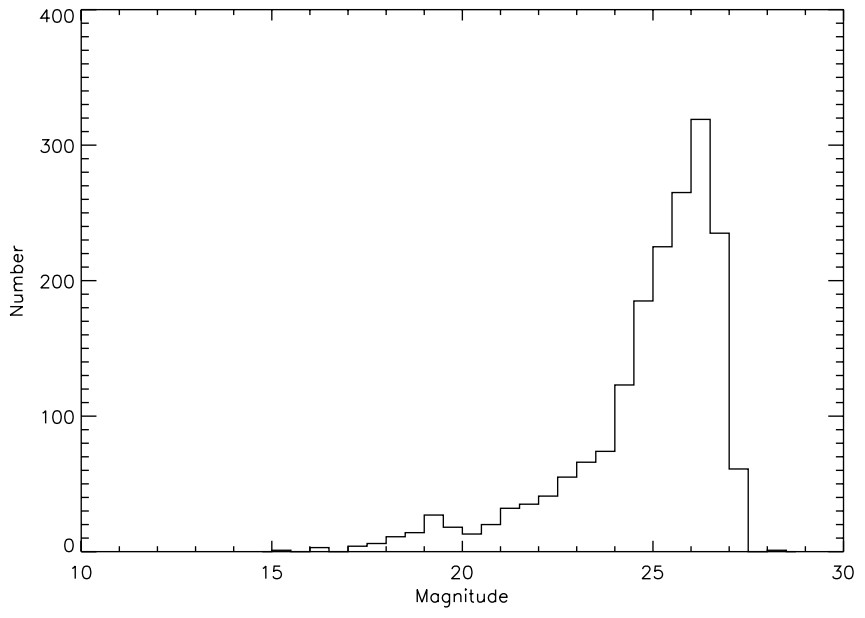

FIg. 1.-Distribution in magnitudes of galaxies in an $R$-band image of Abell 1689. There are two clearly identifiable peaks in this distribution. While the majority of the objects in our sample are faint, with a magnitude peak around 26, there is also a low-magnitude peak at around 19. This corresponds primarily to cluster members, which are concentrated toward the center of the images, and bright stars in the field.

Using the weighted moments necessitates two corrections to equation (14). The first of these corrections results from the fact that there is a difference in the centroid shift between the weighted and unweighted calculations. The second correction has to do with the fact that the total flux is not conserved by lensing. Ordinarily, this is related by the Jacobian of the coordinate transformation; however, when using a window function, the transformation must be considered explicitly.

Incorporating these corrections, we can write

$$
\hat{\mathcal{M}}=\mathcal{M}\left(\hat{Q}_{i j}, \ldots\right)+\Delta \mathcal{M}
$$

where $\Delta \mathcal{M}$ is a $4 \times 4$ matrix representing the correction terms. These terms are proportional to sums of $Q_{i j k l m n}$ and $Q_{i j} Q_{k l m n}$. The reader is referred to GL07 for details regarding the computation of these terms, which are expressed in full in the Appendix to that paper and available as an IDL function from the flexion Web site. ${ }^{5}$

\section{DATA AND PROCESSING PIPELINE}

\subsection{Data}

Our data consists of 20 HST ACS images of Abell 1689, taken using the Wide Field Camera (WFC) during HST cycle 11 by H. Ford. These images are all 2300-2400 s exposures covering a square field of view of $3.4^{\prime}$ on each side (corresponding to $\sim 460 h^{-1} \mathrm{kpc}$ at the distance of the cluster) and are described in detail in Broadhurst et al. (2005b). Of these images, four were taken using the F475W filter ( $G$ band), four using the F625W filter $(R$ band), five using the F775W filter ( $I$ band), and seven using the F850LP filter ( $Z$ band).

Because of the sensitivity of the ACS camera, these exposures resolve objects down to a magnitude of $\sim 27$ in the $R$ band, which makes them ideal for looking at very faint background sources. In addition, this sensitivity gives rise to a very high background source count: our final catalog had $\sim 200$ sources $\operatorname{arcmin}^{-2}$. However, in images of Abell 1689, the central cluster galaxies are a dominant feature, with $R$-band magnitudes as low as $15-16$.

\footnotetext{
5 See http://www.physics.drexel.edu/ goldberg/flexion/. 
Figure 1 shows the magnitude distribution for sources in an $R$-band image, and two distinct peaks can be identified. The lower magnitude peak corresponds primarily to central cluster members and bright stars, while the background galaxies are seen in the larger, high-magnitude peak.

The large spread in magnitudes seen in Figure 1 presents several difficulties in the source detection and extraction process. Typical source extraction software packages, such as SExtractor (Bertin \& Arnouts 1996), require various detection and objectsize thresholds to be set prior to source extraction. In images where there is such a large range of brightnesses, setting lenient detection criteria will result in detection of fainter background sources, but will also lead to excessive blending of images in the neighborhood of the brightest sources. On the other hand, a strict set of criteria will reduce the amount of blending seen, but will also favor detection of foreground objects over the background objects that we are interested in. In $\S 3.2 .2$ we discuss the implementation of a two-pass source detection strategy carried out using SExtractor. This technique is modeled on the "hot" and "cold" source detection strategy described by Rix et al. (2004), who first noted that a single set of SExtractor parameters is generally insufficient for the detection of all objects of interest in ACS images.

\subsection{Catalog Generation}

\subsubsection{Coaddition of Images}

For the initial source detection, we coadded our images using the SWarp software package. ${ }^{6}$ This software produces backgroundsubtracted, median-stacked images, which allows us to clean our images of spurious hotspots and bad pixels. Images are read in by the software, and the background is estimated and subtracted out. The images are then resampled and projected into subsections of the output frame using any of a number of astrometric projections defined in the wavelength coincidence statistics (WCS) standard. Of the projections included in the software, we opted to use the distorted tangential (gnomonic) projection. This projection introduces very little distortion in images smaller than $10^{\circ}$ (see Goldberg \& Gott 2007) and is recommended in the SWarp software manual for small fields. The images are then coadded by taking the median value at any given location.

In addition to cleaning our images of bad pixels, stacking the images increases the signal-to-noise ratio within a given image, thus better enabling us to detect faint background sources. A single stacked image was generated in $G, R$, and $I$ bands. In the $Z$ band, we opted to create two stacked images. Our initial data set consisted of seven exposures in this band. For the purposes of source detection, we found that very little advantage was gained by stacking seven images compared to stacking three or four frames. Thus, we created two $Z$-band images, comparisons between which provide an important test of the effectiveness of our source detection and extraction strategy.

\subsubsection{SExtractor Two-Pass Strategy}

We use SExtractor (Bertin \& Arnouts 1996) to identify and extract sources in our images. This software allows the user to specify a number of input and output parameters, most notably a detection threshold and a minimum number of connected pixels required to be considered an object. In addition, the software is able to produce background and variance maps for the input images and to generate background-subtracted frames.

As noted above, a single SExtractor run will generally result in an incomplete catalog of background sources. Thus, source ex-

\footnotetext{
${ }^{6}$ See http://terapix.iap.fr/soft/swarp.
}

traction is carried out in two stages. The first stage is designed to detect only foreground objects by employing the cross identification utility included in the software. This utility allows the user to supply a catalog of object positions and to instruct the software to either include or exclude the sources in the catalog. We supplied the locations of known foreground objects compiled from the list of spectroscopically confirmed cluster members presented by Duc et al. (2002), prominent stars identified by eye, and objects identified as cluster members by the NASA/IPAC Extragalactic Database (NED). For this pass, we set the detection threshold at $4 \sigma$ and the minimum area of detected objects at 10 pixels.

During this first run, background and variance maps were generated, and this information was used to mask out the foreground objects in order to simulate an emptier field. A friends-of-friends algorithm was used to identify connected pixels above our detection threshold at the locations of the foreground objects. These pixels were then masked out according to $f_{\text {pix }}=f_{\text {bkg }}+\mathcal{R} \sigma_{\text {pix }}$, where $\mathcal{R}$ is a random number drawn from a standard normal distribution $(\mu=0, \sigma=1), \sigma_{\text {pix }}$ is the standard deviation of the background measured at the pixel location, and $f_{\text {bkg }}$ is the background level. Thus, the foreground objects are replaced with a simulated noisy background which has the same statistical properties as the background measured in that region of the image.

A secondary SExtractor run was then performed on the masked image, using less stringent detection criteria (detection threshold: $2 \sigma$; minimum area: 15 pixels) to pick out background galaxies in the field. This strategy alleviated any blending problems we might otherwise have had by removing foreground objects prior to lowering the detection threshold. In addition, it removed from our catalog known foreground objects which, if included in the subsequent analysis, could dilute the measured shear and flexion signals.

A final catalog of background objects was generated by comparing the image detections across different bands. An object was included in the catalog if it was detected in at least two different bands, one of which was either the $R$ or $I$ band.

Whilst the stacked images allow us to better identify legitimate background sources, they are generally not suitable for carrying out measurements, particularly of shear. This is because the PSF varies from image to image; each exposure is offset slightly from the others, and this results in a very complicated PSF in the stacked image that is impossible to model simply. For this reason, the shapelet measurements were carried out on the unstacked images, allowing an explicit PSF deconvolution to be carried out during the shapelet analysis.

It was thus necessary to run SExtractor on the individual frames, since the shapelet parameters used in the analysis depend on SExtractor measurements of galaxy shapes in a given image $\left[\theta_{\max }=1.5\left(a^{2}+b^{2}\right)^{1 / 2}\right]$. We used the two-pass strategy described above, using detection thresholds of $1 \sigma$ less than those used on the stacked images to reflect the lower signal-to-noise ratio in the individual frames (the minimum source areas remained the same). In addition, in the second pass, we required that SExtractor detect only those objects that were detected in the stacked frames and included in the master background object catalog. This requirement avoided any spurious detections resulting from bad pixels within the individual exposures.

\subsection{Shapelet Analysis and Correction of Image Distortions}

Source extraction was carried out on each individual frame, generating a catalog for that frame that contained information about the size, shape, and magnitude of each detected object. The 

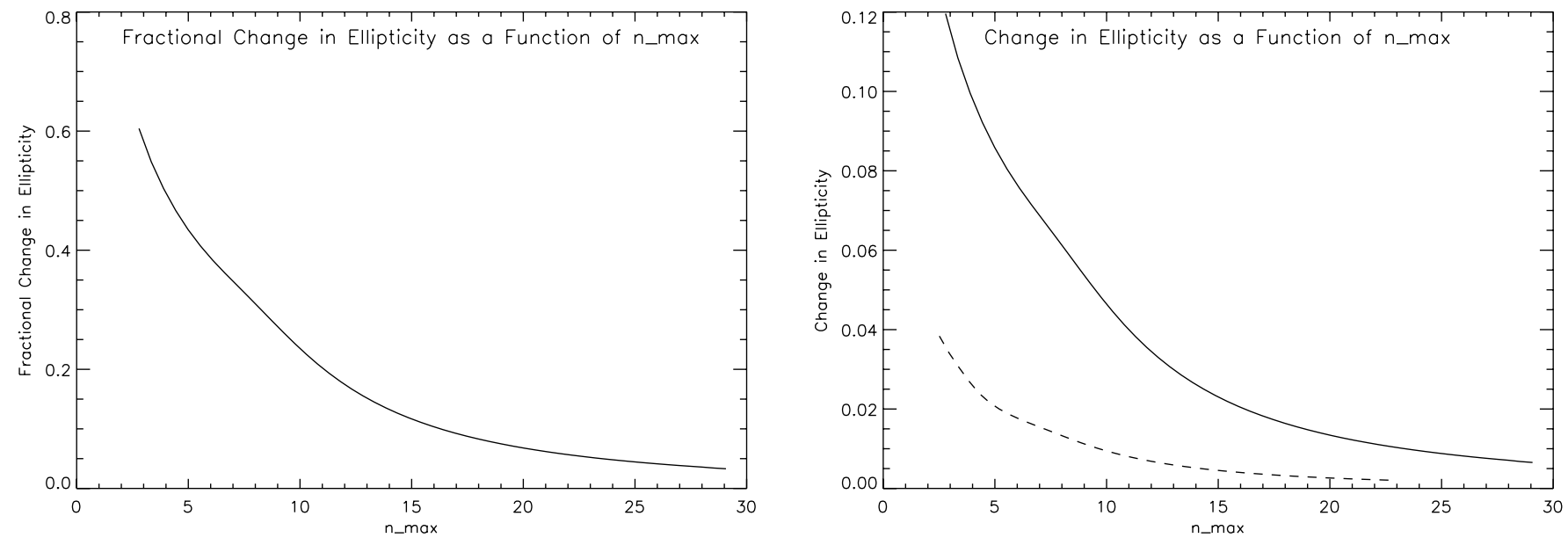

FIG. 2. Left: Fractional error in the ellipticity induced by the PSF plotted against $n_{\max }=\left(\theta_{\max } / \theta_{\min }\right)-1$ for a series of test galaxies with an elliptical Gaussian profile with $|\epsilon|=0.2$. Right: Comparison of the absolute change in ellipticity for a circular source (dashed line) and an elliptical source with $|\epsilon|=0.2$ (solid line).

next task was to generate a postage stamp of each individual galaxy and perform a shapelet decomposition in order to measure the shear and flexion.

To generate a postage stamp, a circular region around each galaxy image was extracted. The size of this region was determined by the SExtractor estimate of the size of the galaxy. A segmentation map was then created by identifying all pixels belonging to the primary source, as well as any other sources found within the extracted region. At this stage, if the primary source was found to extend close to, or beyond, the boundary of the postage stamp, the size of the extracted region was increased appropriately. The segmentation map was then checked for blending of the primary source. Where there was no blending, any other sources in the region were masked out, using a similar procedure to that used in masking out the foreground objects. Postage stamps in which blending of the primary source was found were automatically rejected.

The next step in the process is a shapelet decomposition and PSF deconvolution. As discussed in GL07, the computing time required for a shapelet decomposition scales roughly as $\theta_{\max }^{4}$ (not including a PSF deconvolution, which slows the process further). This means that for very large objects, the decomposition time becomes prohibitive. GL07 discuss a method for speeding up the decomposition in larger objects by regridding them into larger pixels. This method works adequately; however, it does not take into consideration any PSF effects. We discuss the effect of the PSF on shear and flexion measurements below and describe our strategy for minimizing the shapelet analysis time without significant reduction in the accuracy of our shape measurements.

\subsubsection{PSF Modeling Using TinyTim}

We modeled the PSF using the TinyTim software package (Krist 1993). This software was designed to simulate the PSF of the WFC using a three-step process that allows it to account for variations in the PSF due to chip location and filter wavelength. We used the default WFC settings for the focus and PSF size in our TinyTim simulations.

The ACS wide field camera has a well-studied geometric distortion in its images, which is a result of the off-axis location of the camera on HST. This distortion has been modeled (Meurer et al. 2003) and is well fit by a fourth-order polynomial. TinyTim attempts to take this distortion into account by applying it to the PSF in the third stage of the PSF generation.
This is necessary when correcting for the PSF at the point of parameter estimation. However, our shapelet technique involves an explicit PSF deconvolution before any shape measurements are made. Including this correction to the PSF prior to deconvolution does not amount to correcting the image for the geometric distortion. Thus, we did not include this step in our PSF generation; rather, we used an undistorted PSF model in the deconvolution. At the point of parameter estimation, we applied a correction for the geometric distortion as described in $\S 3.4$.

As the decomposition time in larger objects is of concern, one might ask what effect this model PSF has on shear and flexion measurements. The flexion measured in the PSF is small (typically
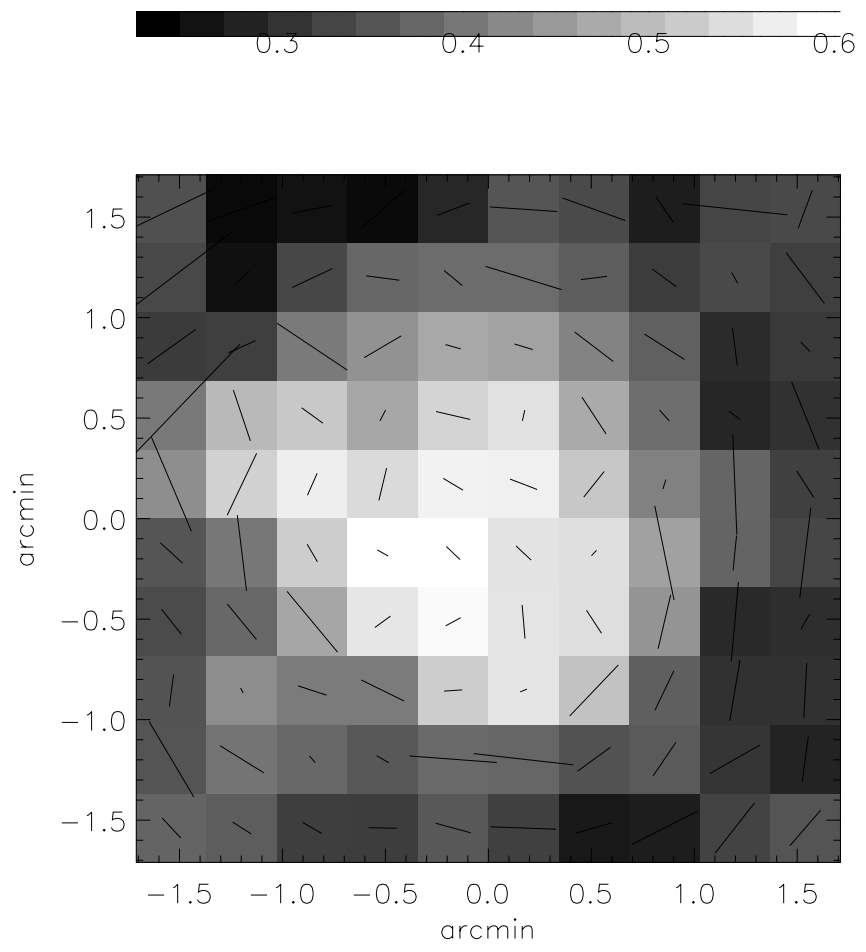

FIG. 3.-Weak lensing shear field along with a nonparametric reconstruction for ACS images of Abell 1689. Note that the overall normalization of the reconstruction is subject to uncertainty due to the mass-sheet degeneracy in a finite field. North points to the bottom right of the image, and the width of each bin corresponds to approximately $45 h^{-1} \mathrm{kpc}$ at the distance of the cluster. 


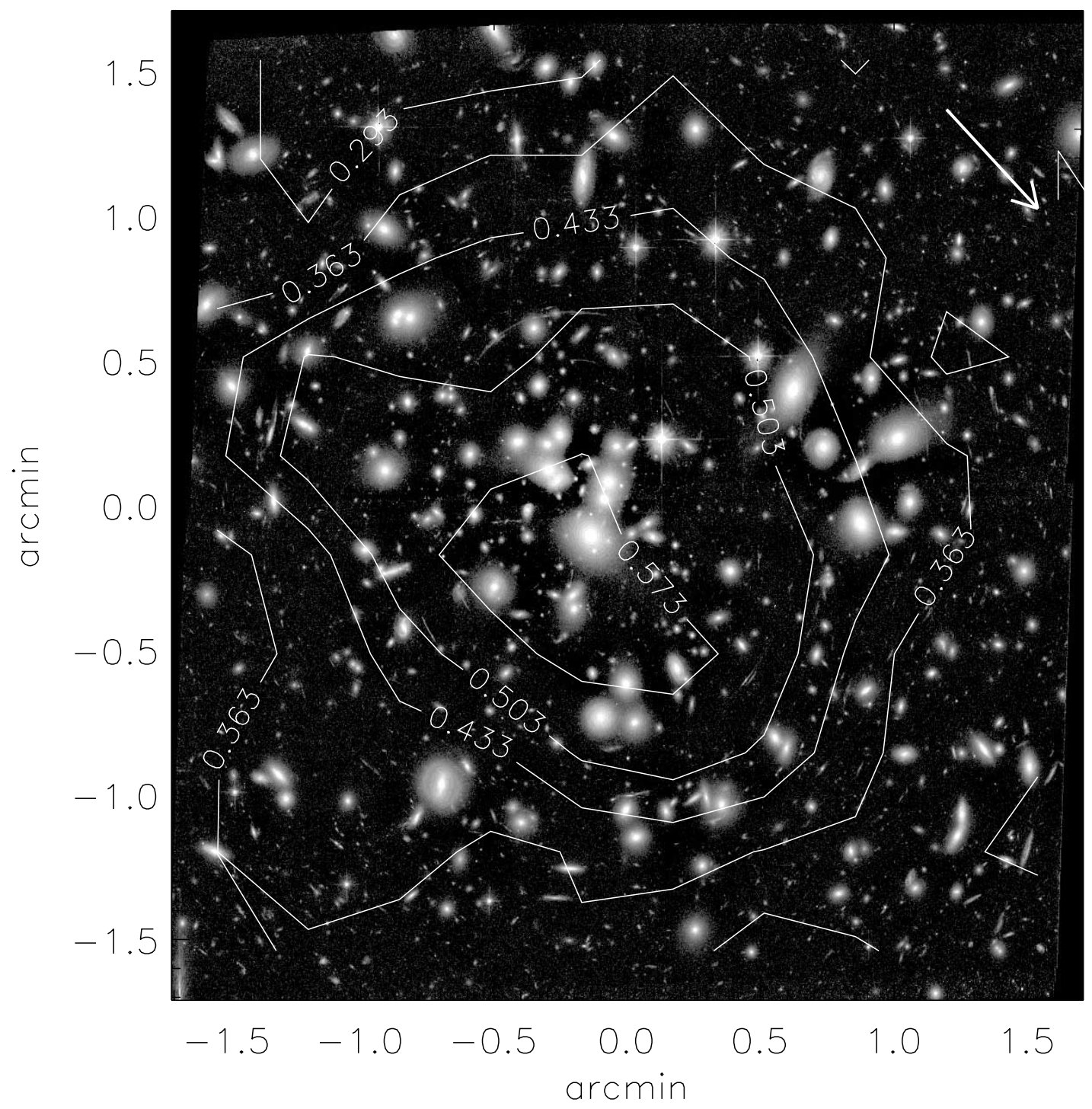

FIG. 4.-Contour plot of the convergence $\kappa$ determined using the shapelet measurements of the ellipticity of background sources in the field. The contours clearly identify small concentrations of foreground galaxies within the cluster.

of order $10^{-4}$ to $10^{-5}$ pixel $^{-1}$ ). This will induce a flexion in the image which scales roughly as

$$
\mathcal{F}_{\text {ind }} \sim \mathcal{F}_{\mathrm{PSF}} \frac{a_{\mathrm{PSF}}^{4}}{a_{\mathrm{src}}^{4}+a_{\mathrm{PSF}}^{4}},
$$

where $a_{\mathrm{PSF}}$ and $a_{\mathrm{src}}$ refer to the semimajor axis of the PSF and the source, respectively. This scaling relation follows from equations (43) - (45) in GL07 and is discussed in more detail in the Appendix. Clearly, this drops off very rapidly with increasing source size; thus, the PSF will be a subdominant effect in measurements of flexion for images with a semimajor axis comparable to that of the PSF, which, for a typical $R$-band simulation, is found to be $a_{\mathrm{PSF}} \simeq 2.0$ pixels. Thus, the flexion measurements, in general, do not need to be corrected for PSF effects, particularly if a minimum size criterion is introduced (as in $\S 3.7$ ).

In order to assess how the PSF affects the measured ellipticities of galaxies, we simulated Gaussian "galaxies" of various sizes and ellipticities, convolved them with a model $R$-band PSF in real space, and computed the change in ellipticity of the source. The ellipticity was computed using the unweighted moments of the light distribution, and we defined the change in ellipticity of the source as

$$
\Delta|\epsilon| \equiv \sqrt{\left(\epsilon_{1}^{(0)}-\epsilon_{1}^{(c)}\right)^{2}+\left(\epsilon_{2}^{(0)}-\epsilon_{2}^{(c)}\right)^{2}},
$$

where the superscripts (0) and (c) refer to the unconvolved and convolved images, respectively.

The left panel of Figure 2 shows the fractional change in ellipticity, $\Delta|\epsilon| /|\epsilon|$, plotted as a function of $n_{\text {max }}$ (defined in $\S 2.2 .1$ ) for an elliptical source with $|\epsilon|=0.2$. We also show in Figure 2 a comparison of the absolute change in ellipticity for a circular and an elliptical source.

From Figure 2 we can see that the effect of the PSF on the ellipticity of the source drops to below $10 \%$ at $n_{\max }=20$. Thus, we defined a "large object cutoff"; any object with an $n_{\max }>20$ was considered to be sufficiently large that PSF effects do not dominate, and an explicit deconvolution was not carried out. In addition, as described in GL07, images with $n_{\max }>50$ were regridded into larger pixels of size $n_{\max } / 40$ in pixel units in order to further reduce the decomposition time for very large objects. In order to 
exclude objects for which the shapelet fit was not successful, we rejected any sources for which the decomposition had a $\chi^{2}$ per degree of freedom that was greater than 2.0.

\subsection{ACS Geometric Distortion Correction}

As described above, the ACS WFC induces a geometric distortion in images that is well fit by a fourth-order polynomial (Meurer et al. 2003),

$$
\begin{aligned}
& x^{\prime}=\sum_{i=0}^{4} \sum_{j=0}^{i} a_{i j} x^{j} y^{i-j}, \\
& y^{\prime}=\sum_{i=0}^{4} \sum_{j=0}^{i} b_{i j} x^{j} y^{i-j},
\end{aligned}
$$

where the primed coordinates refer to the undistorted frame, the unprimed coordinates are the distorted pixel coordinates relative to the central pixel in each chip, and $a_{i j}$ and $b_{i j}$ are the bestfit coefficients and are specific to each of the two chips on the ACS camera. The fit parameters can be found in the TinyTim software package.

We can use these transformations to evaluate the $\mathbf{A}$ and $\mathbf{D}$ operators defined in equations (2) and (3). This allows us to evaluate the shear and flexion induced by the geometric distortions as a function of location on the chip. These induced distortions can then be subtracted from the measured shear and flexion.

We found that the mean magnitude of the induced ellipticity across the field of view was significant, with a mean of $\overline{|\gamma|}=$ 0.0405 and a standard deviation of $\sigma_{|\gamma|}=0.0083$. The induced flexion, however, was found to be negligible over the entire field of view, with $\overline{|\mathcal{F}|}=3.05 \times 10^{-4}$ pixel $^{-1}, \overline{|\mathcal{G}|}=1.00 \times$ $10^{-4}$ pixel $^{-1}, \sigma_{|\mathcal{F}|}=4.61 \times 10^{-5}$ pixel $^{-1}$, and $\sigma_{|\mathcal{G}|}=2.34 \times$ $10^{-5}$ pixel $^{-1}$.

In light of this, only the measured ellipticity was corrected for the geometric distortions. In addition, since the PSF was found to have little effect on the flexion for images with $a \simeq 2.0$, we opted to measure the flexion on the stacked images, for which the signalto-noise ratio is higher, and incorporated a lower limit on the size of images analyzed. We note that, as a result of the reprojection of the images during the stacking process, the geometric distortions are reduced in the stacked frames.

\subsection{Nonparametric Convergence Map Generation}

Each background galaxy had from 1 to 20 independent ellipticity estimates, depending on how many of the fields it was detected in. For those detected at least twice, we estimated the mean and measurement error of the galaxy ellipticities via the relation

$$
\sigma_{\text {meas }}=\frac{\left\langle\left(\varepsilon_{i}-\bar{\varepsilon}\right)^{2}\right\rangle^{1 / 2}}{\sqrt{N-1}}
$$

The mean and error were actually measured iteratively, and all measurements outside of $2.5 \sigma$ of the mean were discarded as outliers. An ellipticity error was then assigned to each galaxy as a quadratic sum of the measurement uncertainty and 0.3 , the intrinsic standard deviation in ellipticities.

We then binned the field into cells approximately $20^{\prime \prime}$ on a side with an estimated ellipticity given by the weighted mean of the measurements in the individual frames and with bin errors estimated using standard error propagation. Various rejection criteria in the pipeline (most notably blended sources and those with poor shapelet reconstructions or fewer than two detections) resulted in a

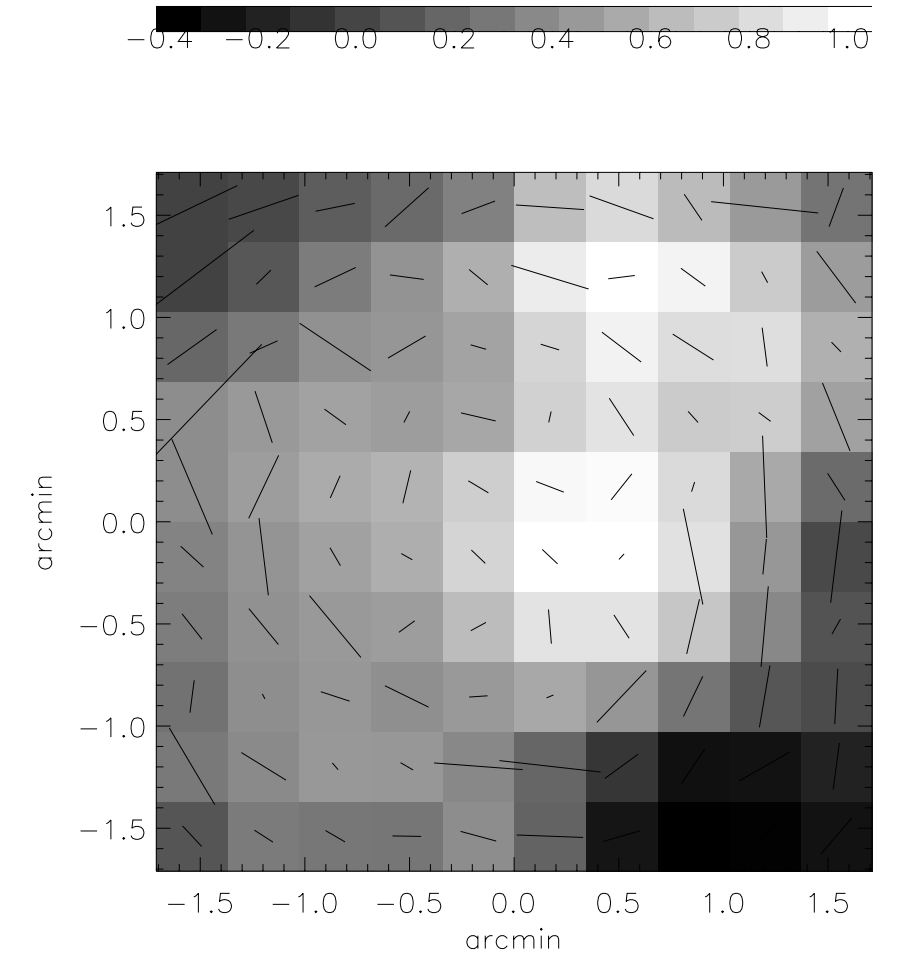

FIG. 5.-Shear map and the $\kappa$-field found using both shapelet measurements of the ellipticity and multiple-image pairs identified by L06. In this plot, the width of each bin corresponds to $45 h^{-1} \mathrm{kpc}$ in the cluster plane.

reduced source count of $88 \operatorname{arcmin}^{-2}$, which is much higher than that typically found in weak lensing studies of this cluster (see, e.g., Broadhurst et al. 2005a).

We then applied the finite inversion technique advocated by Seitz \& Schneider (1995). They show that the density field can be solved iteratively (up to the mass-sheet degeneracy) as

$\kappa(\boldsymbol{\theta})-\kappa_{0}=\frac{1}{\pi} \int d^{2} \boldsymbol{\theta}\left[1-\kappa\left(\boldsymbol{\theta}^{\prime}\right)\right] \mathcal{R}\left[\mathcal{D}^{*}\left(\boldsymbol{\theta}-\boldsymbol{\theta}^{\prime}\right)\langle\epsilon\rangle\left(\boldsymbol{\theta}^{\prime}\right)\right]$,

where the convolution function $\mathcal{D}$ can be written as

$$
\mathcal{D}(\boldsymbol{\theta})=-\frac{\left\{1-\left[1+\left(\theta^{2} / \theta_{s}^{2}\right)\right] \exp \left(\theta^{2} / \theta_{s}^{2}\right)\right\}}{\left(\theta_{1}-i \theta_{2}\right)^{2}},
$$

and our smoothing scale $\theta_{s}$ was set to half a bin size.

The above method produces a reasonable mass reconstruction (see $\S 4$ ); however, a better constrained mass model can be derived from combining both weak and strong lensing methods (see, e.g., Kneib et al. 2003; Smith et al. 2005; Bradac et al. 2005a, 2005b, 2006 ). In order to do this, we follow the method of Bradac et al. (2005a). Our multiple-image catalog is taken from L06, and we assume that $D_{l s} / D_{s}=0.625$, i.e., the mean redshift of the background sources is 0.9 , as in L06. Note that applying the Bradac et al. technique with only weak lensing constraints produces a very similar reconstruction to that found using the approach of Seitz \& Schneider (1995).

\subsection{Parametric Convergence Map Generation}

Flexion typically dominates the lensing signal on a much smaller scale than shear. Thus, the binning scale described above is not appropriate for flexion measurements, as the signal would be entirely dominated by shot noise. In this context, discrete estimates of 


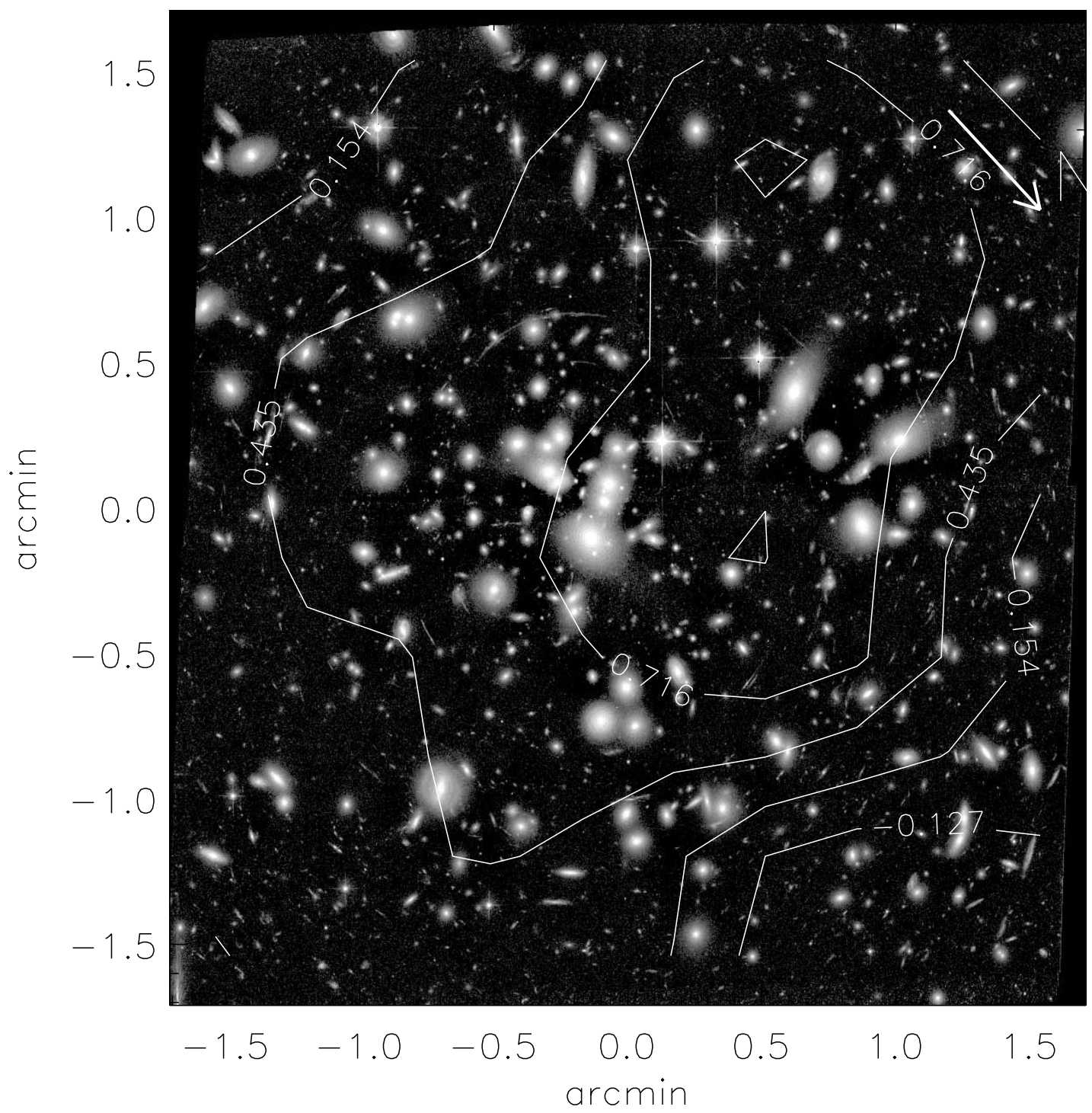

Fig. 6.-Contour plot of the convergence $\kappa$ determined using the shapelet measurements of the ellipticity of background sources in the field combined with strong lensing data from L06.

the flexion of individual galaxies (parametric reconstructions) are in order.

As the flexion was measured on the stacked images, each source had up to five flexion measurements. We included only those objects measured in at least three of the five stacked frames and combined the flexion estimates using a similar iterative statistic to that described above for shear. In addition, we only included objects that had a semimajor axis larger than $0.12^{\prime \prime}$ (2.4 pixels), which were in the brightest $90 \%$ of the sample of background objects (which still yielded approximately 75 galaxies $\operatorname{arcmin}^{-2}$ ), and which had absolute values of flexion that were smaller than 4 times the random scatter, to exclude extreme outliers. We found that the second flexion produced significantly higher scatter than the first flexion, and thus, the first flexion was the only one used in our analysis.

To generate a convergence map, we modeled each cluster galaxy under the assumption that it is a singular isothermal sphere with the flexion profile given in BGRT06,

$$
F_{E}(\theta)=\frac{-4 \pi\left(\sigma_{v} / c\right)^{2}}{2 \theta^{2}} .
$$

The flexion data was used to fit $\sigma_{v}^{2}$ for each foreground galaxy for which at least three background sources were identified with an angular separation of $\leq 10^{\prime \prime}$. Out of our initial sample of 62 foreground galaxies, only 36 met this criterion, and thus, our final mass reconstruction was based on the fits obtained for these 36 galaxies.

Since this is a noisy measure, some estimators predicted a negative value of $\sigma_{v}^{2}$. These were retained in our map in order to keep the convergence estimate unbiased. The mean velocity dispersion computed this way was found to be $\left\langle\sigma_{v}\right\rangle=321 \mathrm{~km} \mathrm{~s}^{-1}$. After estimating the velocity dispersions, a density field was laid down,

$$
\kappa(\boldsymbol{\theta})=\sum_{i} 2 \pi\left(\frac{\sigma_{v, i}^{2}}{c^{2}}\right) \frac{1}{\left|\boldsymbol{\theta}_{i}-\boldsymbol{\theta}\right|},
$$

and then smoothed using a Gaussian kernel on a scale of $10^{\prime \prime}$.

\subsection{Galaxy-Galaxy Flexion Measurements}

We also used our flexion measurements in a galaxy-galaxy ensing study. Galaxy-galaxy measurements of shear are extremely 


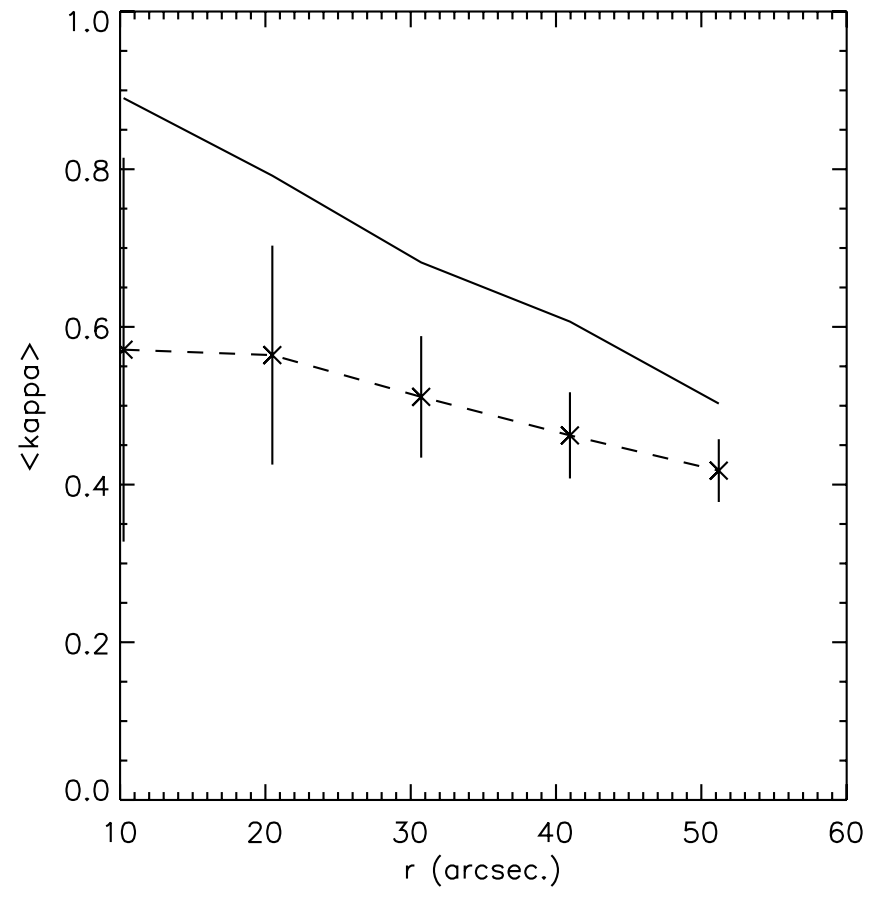

FIG. 7.-Circularly averaged convergence as a function of distance from the center of the cluster from weak measurements alone (dashed line) and from the combined weak + strong calculation (solid line). Clearly, the weak + strong profile shows a much steeper slope than the weak profile. However, there is very good agreement between the two profiles at large radii. The error bars shown in these plots are taken from $\sqrt{N}$ statistics and, thus, are only an approximation.

difficult in cluster environments, since a single source may be partially lensed by many different foreground objects. In addition, the shear signal tends to be dominated by the smooth component of the cluster mass distribution, rather than the substructure. The flexion signal, however, drops off much more quickly with separation and, thus, will almost always be produced by a single source.

We carried out a pairwise comparison between each of the background images and the 62 cluster members in our foreground object catalog. For each, we considered the relative orientation angle and estimated " $B$ " and " $E$ " field flexion signals via the relationships

$$
\begin{gathered}
\mathcal{F}_{E} \equiv \mathcal{F}_{1} \cos (\phi)+\mathcal{F}_{2} \sin (\phi), \\
\mathcal{F}_{B} \equiv-\mathcal{F}_{1} \sin (\phi)+\mathcal{F}_{2} \cos (\phi),
\end{gathered}
$$

where $\phi$ is the position angle of the background source with respect to the foreground galaxy. Lensing naturally gives rise to $\mathcal{F}_{E}<0$ and $\mathcal{F}_{B}=0$; thus, the scatter in the measured $B$-mode signal gives an estimate of the noise in the measurements.

For each, we estimated an uncertainty in the flexion via the relation

$$
\sigma_{F}^{2}=\left(\frac{0.029}{a}\right)^{2}+\sigma_{\text {meas }}^{2}
$$

where the former term on the right-hand side represents the scatter in intrinsic flexion (see GL07) and the latter represents the measurement error as determined from the scatter between frames. Weighting each data point by its signal-to-noise ratio, we computed the average flexion signal as a function of lens-source separation.

Finally, we performed least-squares fitting to an assumed isothermal sphere profile as described in equation (24). Other fits could be done, of course, but the isothermal model was both sim-

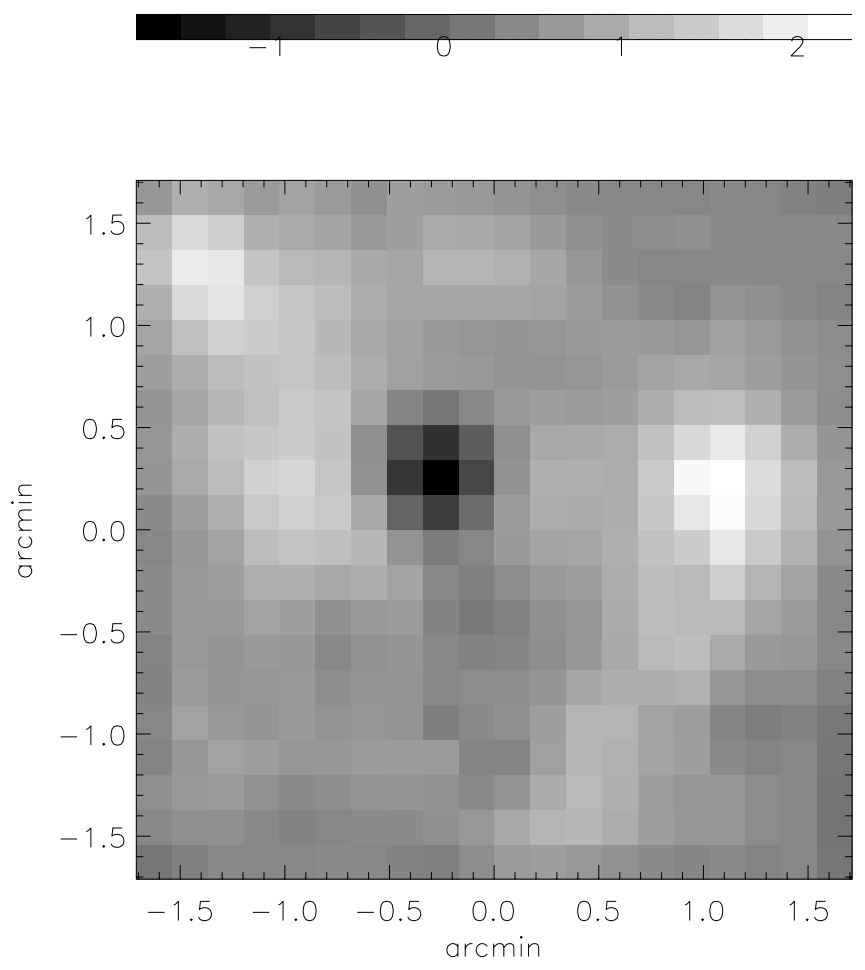

FIG. 8.-Binned convergence found using a parametric model for the cluster galaxies from flexion data. The width of each bin is approximately $22.5 h^{-1} \mathrm{kpc}$ in the cluster plane. Note that a flexion-only reconstruction is sensitive to localized substructure but not smooth gradients in the mass distribution.

ple and seemed to fit the data well. Note that, in this case, we are considering the average signal over all foreground-background pairs, rather than fitting each individual foreground galaxy.

\section{RESULTS AND DISCUSSION}

\subsection{Weak Shear Mass Reconstruction (Nonparametric)}

Most mass reconstructions of Abell 1689 either have been parametric (e.g., L06; Halkola et al. 2006; Broadhurst et al. 2005b; King et al. 2002a), have measured circularly averaged shears (e.g., L06; Bardeau et al. 2005; Broadhurst et al. 2005a; Umetsu et al. 2005), or have been nonparametric reconstructions using strong lensing data (e.g., Diego et al. 2005b). Thus, it is interesting to compare weak nonparametric reconstructions to the above methods to verify that they produce consistent results. This would also lend fuel to the recent efforts to unify weak and strong lensing studies in order to further reduce uncertainty.

In order to generate radially averaged shear profiles of the cluster, the authors mentioned above have made use of ground-based data, either exclusively or as a supplement to the ACS images of the cluster. Part of the reason for this is that the core of the cluster is quite clumpy, and thus, a centroid cannot be uniquely defined. That said, at a distance of $100^{\prime \prime}$ from the brightest cluster member, we find a tangential shear of about 0.2 , in excellent agreement with the weak lensing study of L06.

We have produced a simple shear field as described in $\S 3$ and used this field to reconstruct the density field via the Seitz \& Schneider (1995) smoothed finite-inversion technique. Our shear field and reconstructed $\kappa$-field can be seen in Figure 3 .

With so much attention given to the ability of strong lensing to pick out substructure in clusters, it is gratifying to note that weak lensing alone can identify large concentrations of galaxies. A contour plot of the projected surface mass density, $\kappa$, is shown in Figure 4 , overlaid on an image of the cluster itself. 


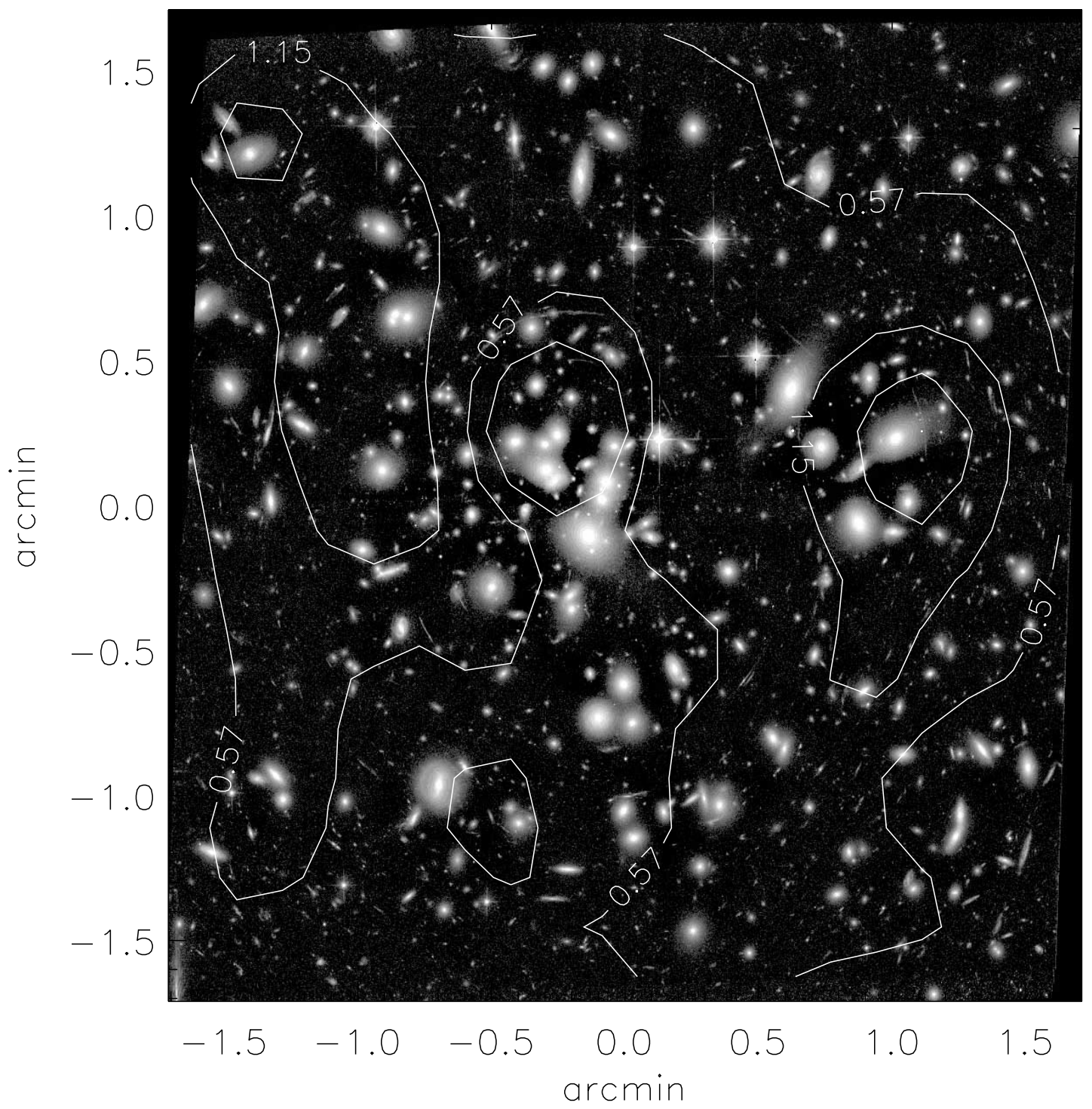

FIG. 9.-Contour plot of the convergence calculated using a parametric model for the cluster galaxies from flexion measurements. This reconstruction shows significant substructure corresponding to the locations of small clumps of cluster galaxies.

For this cluster, we find $\Sigma_{\text {crit }} \simeq 5.7 \times 10^{15} h M_{\odot} \mathrm{Mpc}^{-2}$ (taking $z=0.18$ ), which allows us to estimate the true surface mass density up to the mass-sheet degeneracy. However, since we have not independently measured the shear field far from the center of the cluster, we rely on the estimates of others. L06, for example, found a mean $\bar{\kappa}$ of 0.48 within $1^{\prime}$ of the center of the cluster. We have thus set our mass sheet at a slightly lower threshold, so that the mean over the entire chip is 0.4 .

We note that the density profile is quite shallow, however. We find that a power-law profile is well fit if $\alpha=-0.19$. Over a similar range, L06 fit to a profile with $\alpha \simeq-0.6$. L06, however, base their fit on extrapolation from the slope of the shear outside the ACS image. Inspection of Figure 11 in their work suggests that the circularly averaged profile within the central arcminute may be much shallower.

\subsection{Strong + Weak Lensing Mass Reconstruction (Nonparametric)}

We have combined strong lensing information from L06 with our shear measurements to generate a combined convergence map, following the method of Bradac et al. (2005a). A convergence map and a contour plot of the convergence found by this method can be seen in Figures 5 and 6.

Figure 6 clearly shows an elongation in the direction of the secondary dark matter clump described in L06, which is not seen in Figure 4. Figure 7 compares the circularly averaged convergence as a function of distance from the center of the cluster for the weak and strong+weak mass reconstructions. The strong+weak profile has a much steeper slope than the weak profile. Indeed, we find $\alpha=-0.29$ for the profile shown in Figure 7 .

This difference in slope could result from the fact that our masking scheme results in an underdensity of background sources in the central region of the cluster. This shortage of data points results in a lower shear signal in the central region, thus lowering the computed value of $\kappa$. The underdensity of sources in the central region is found to be an important factor in the errors associated with our parametric flexion reconstruction and is discussed in more detail in $\S 4.3$.

\subsection{Flexion Mass Reconstruction (Parametric)}

A parametric mass reconstruction was generated using (first-) flexion data alone. In dense systems like clusters, we have found 


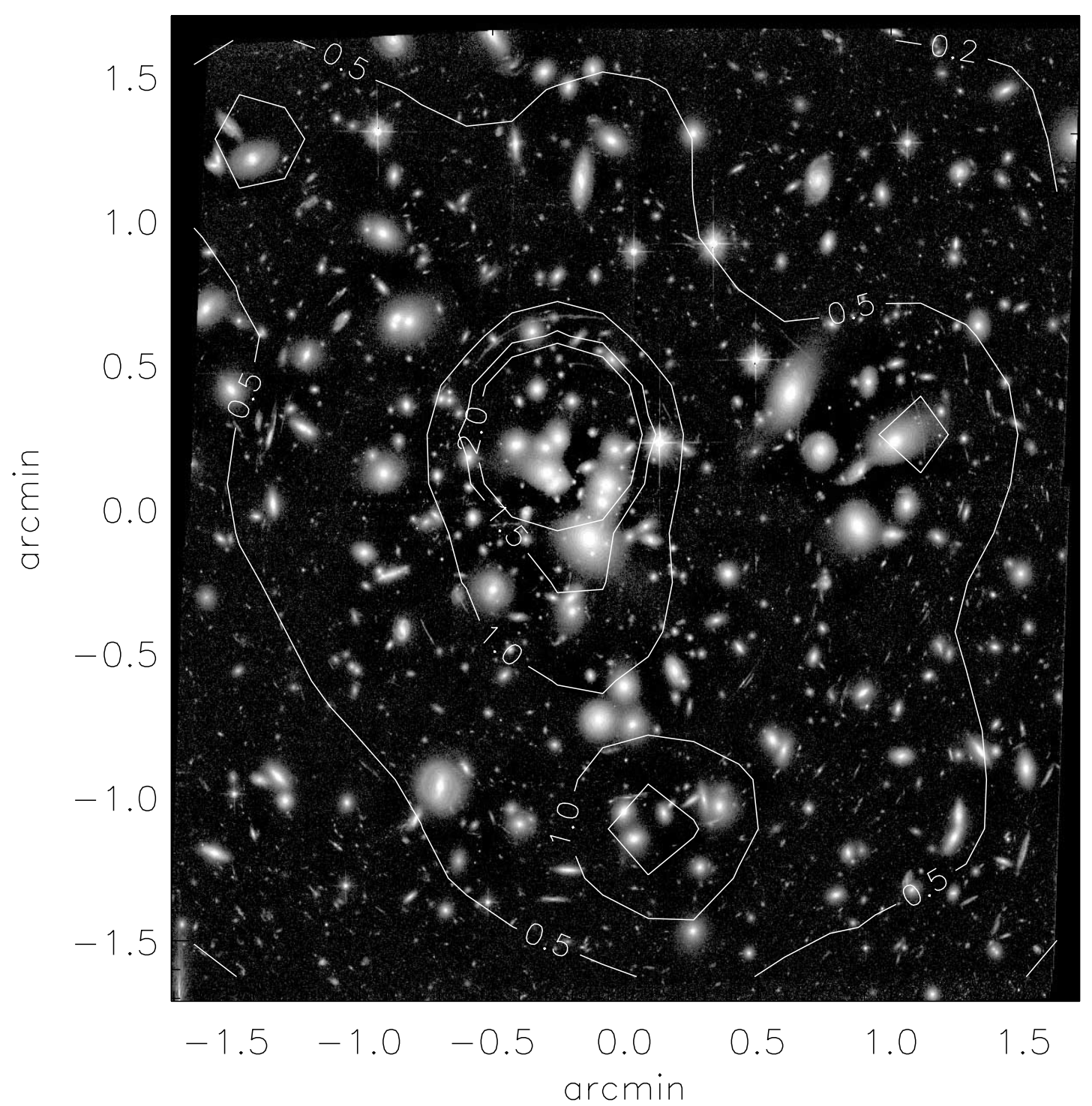

FIG. 10.-Contour plot of the approximate errors in the parametric flexion mass reconstruction. It is evident from this plot that the reconstruction is entirely dominated by noise in the center of the image.

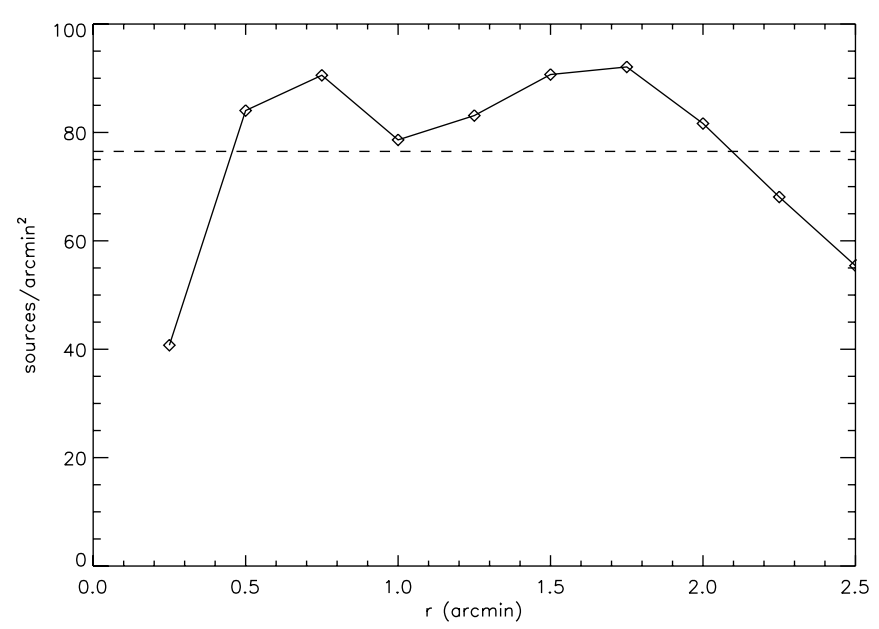

FIG. 11.-Number density of background sources used in our flexion study (averaged over a circular region) plotted as a function of distance from the center of the image. The dotted line shows the average number density over the entire image. There is an apparent downturn in the number density at large $r$. This downturn occurs simply because we reach the edge of the field. that the HOLICs technique is less susceptible than shapelets to contamination by light from the extended wings of lens galaxies and other neighboring sources. Flexion probes the higher order shape moments, so even a small contamination near the edges of postage stamps can cause a significant spurious flexion signal. We thus used our HOLICs measurements exclusively to estimate the flexion signal.

The reconstructed convergence is plotted in Figure 8, and a contour plot is shown in Figure 9. The reconstruction shows significant substructure, which appears to be well correlated with small clumps of galaxies outside the center of the cluster. However, there is a rather worrying underdensity seen in the center of the cluster. In order to asses the significance of this underdensity, it is necessary to quantify the errors associated with the convergence map.

Figure 10 shows the approximate errors in the flexion reconstruction. These errors were computed as follows. Each flexion data point was rotated by an angle drawn from a uniform random distribution in the range $[0,2 \pi]$. Parametric reconstructions were generated using this randomized data, and this procedure was carried out for 1000 randomizations. The errors presented in Figure 10 are the rms values found for each bin. 


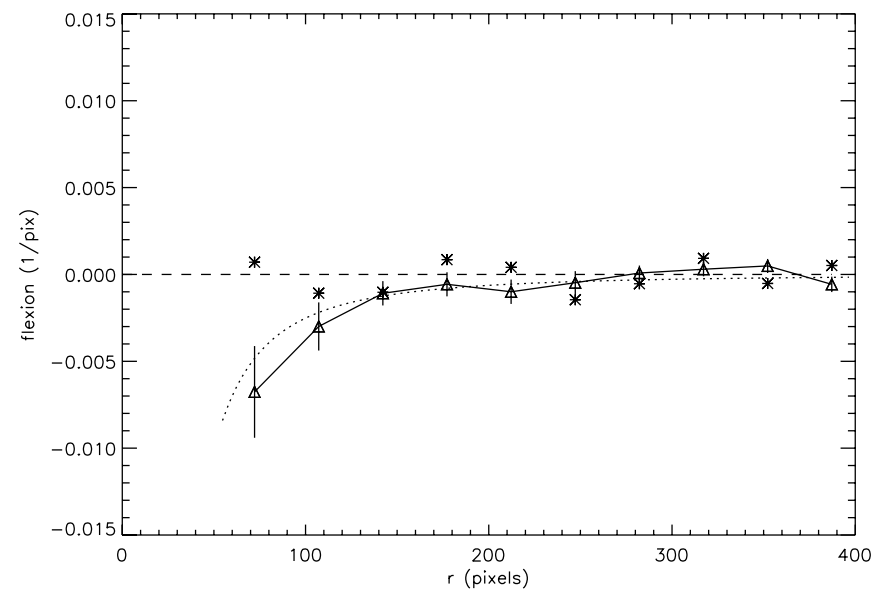

Fig. 12.-Mean galaxy-galaxy flexion signal of cluster galaxies. The $B$-mode signal is plotted as unconnected points and is consistent with zero. The dotted line represents the expected signal for an isothermal sphere with a velocity dispersion $\sigma=295 \mathrm{~km} \mathrm{~s}^{-1}$.

Clearly, the underdensity seen in the center of the convergence map in Figure 8 should be considered as resulting from noise, rather than a real feature of the cluster, as the central region appears to be entirely noise dominated. This is most likely due to the fact that there are fewer background sources found in this region. Figure 11 shows the average number density of background sources plotted as a function of the radius over which this number density is averaged.

The shortage of sources in the central region results from our masking scheme and affects our flexion reconstruction in two ways. First, it means that a foreground galaxy in the center of the cluster is less likely to have the required three nearby background sources, and thus, fewer of the central cluster members will be included in the analysis. Second, those that are included will generally be fit using fewer data points than the outlying cluster members, and thus, these fits will have larger associated errors.

Thus, the underdensity seen in the center of the flexion reconstruction should not be believed. However, in the outlying regions of the image, the noise is seen to drop significantly, and the substructures seen in these regions appear to be real features.

\subsection{Galaxy-Galaxy Flexion Signal}

In addition to a large-scale map of the cluster, we have also generated a composite circular profile of the cluster member galaxies via flexion measurements. Figure 12 shows the galaxy-galaxy (first-) flexion averaged over all background-foreground pairs meeting our selection criteria.

We find that the mean cluster member can be fit well by an isothermal sphere with $\sigma_{v}=(295 \pm 40) \mathrm{km} \mathrm{s}^{-1}$. Given the relatively large uncertainty in velocity and the relatively narrow scat- ter in the magnitude of the cluster members $\left(\sigma_{r} \simeq 1\right)$, we are unable to effectively split the cluster members into subgroups. However, we can compare this result to that expected from the Faber-Jackon relation.

The mean absolute magnitude of the sample is approximately -21.5 in the $R$ band. Taking the canonical Faber-Jackson relation

$$
\sigma_{v}=220 \mathrm{~km} \mathrm{~s}^{-1}\left(\frac{L}{L_{*}}\right)^{0.25},
$$

we find an expected velocity dispersion of approximately $390 \mathrm{~km} \mathrm{~s}^{-1}$, in accord with our measurements.

In their Table 3, L06 compute a best-estimate rms for several member galaxies $\left(\sim 200 \mathrm{~km} \mathrm{~s}^{-1}\right)$ and the brightest cluster galaxy $\left(\sim 500 \mathrm{~km} \mathrm{~s}^{-1}\right)$. Our mean flexion estimate falls squarely in the middle of this distribution. We also note that the mean velocity dispersion found when computing the parametric flexion reconstruction $\left(321 \mathrm{~km} \mathrm{~s}^{-1}\right)$ is consistent with that derived from the galaxy-galaxy lensing study, within the error bars of the latter.

\section{SUMMARY}

We have used a shapelet-based shear measurement technique to create a nonparametric mass reconstruction of the galaxy cluster Abell 1689. Using only weak-lensing data, we found significant ellipticity and substructure in the cluster. Combining the weak lensing data with strong-lensing data from L06 improved the resolution of the mass map and increased the slope of the cuspy central density profile. The combined analysis also identified a secondary dark matter clump found by L06. Using an entirely new and independent flexion analysis, we were able to verify the position of this clump, and other substructure, via a parametric reconstruction of the cluster mass, modeling each of the cluster members as a singular isothermal sphere. The substructure observed in the flexion reconstruction is well correlated with the locations of groups of cluster galaxies.

We have also used flexion data to probe the halos of individual cluster galaxies. Using a similar parametric reconstruction, we measure a highly significant $(\sim 13 \sigma)$ galaxy-galaxy flexion signal. In agreement with previous, nonflexion measurements, we find a mean velocity dispersion of $(295 \pm 40) \mathrm{km} \mathrm{s}^{-1}$ for the cluster galaxies.

The authors would like to thank David Bacon, Sanghamitra Deb, John Parejko, Lindsay King, and Marceau Limousin for useful discussions. This work was supported by NASA ATP NNG 05-GF61G and HST Archival grant 10658.01-A. A. L. is supported by a BP/ PPARC Dorothy Hodgkin Postgraduate Award.

\section{APPENDIX}

\section{INDUCED FLEXION DUE TO THE PSF}

From equations $(43)-(45)$ in GL07, we have the relations

$$
Q_{i j k}=Q_{i j k}^{(0)}+P_{i j k}, \quad Q_{i j k l}=Q_{i j k l}^{(0)}+d P_{i j k l},
$$

where

$$
\begin{gathered}
d P_{i j k l} \propto P_{i j k l} \propto a_{\mathrm{PSF}}^{4}, \\
Q_{i j k l}^{(0)} \propto a_{\mathrm{src}}^{4} .
\end{gathered}
$$


Now

$$
\begin{aligned}
\mathcal{F} & \sim \frac{Q_{i j k}}{Q_{i j k l}} \\
& =\frac{Q_{i j k}^{(0)}+P_{i j k}}{Q_{i j k l}^{(0)}+d P_{i j k l}} \\
& =\frac{Q_{i j k}^{(0)}}{Q_{i j k l}^{(0)}} \frac{Q_{i j k l}^{(0)}}{Q_{i j k l}^{(0)}+P_{i j k l}}+\frac{P_{i j k}}{d P_{i j k l}} \frac{d P_{i j k l}}{Q_{i j k l}^{(0)}+d P_{i j k l}} \\
& \sim \mathcal{F}^{(0)} \frac{a_{\mathrm{src}}^{4}}{a_{\mathrm{src}}^{4}+a_{\mathrm{PSF}}^{4}}+\mathcal{F}_{\mathrm{PSF}} \frac{a_{\mathrm{PSF}}^{4}}{a_{\mathrm{src}}^{4}+a_{\mathrm{PSF}}^{4}} .
\end{aligned}
$$

The former term in the above expression will dominate for $a_{\mathrm{src}}>a_{\mathrm{PSF}}$, and the coefficient of the $\mathcal{F}^{(0)}$ term will approach unity as $a_{\text {src }}$ becomes large. In the case of small $a_{\text {src }}$, the term in $\mathcal{F}_{\text {PSF }}$ will become important, provided that $\mathcal{F}_{\text {PSF }}$ itself is significant.

\section{REFERENCES}

Abdelsalam, H. M., Saha, P., \& Williams, L. L. R. 1998, NewA Rev., 42, 157 Bacon, D. J., Goldberg, D. M., Rowe, B. T. P., \& Taylor, A. N. 2006, MNRAS, 365, 414 (BGRT06)

Bardeau, S., et al. 2005, A\&A, 434, 433

Bartelmann, M., \& Schneider, P. 2001, Phys. Rep., 340, 291

Bernstein, G. M., \& Jarvis, M. 2002, AJ, 123, 583

Bertin, E., \& Arnouts, S. 1996, A\&AS, 117, 393

Bradac, M., Schneider, P., Lombardi, M., \& Erben, T. 2005a, A\&A, 437, 39

Bradac, M., et al. 2005b, A\&A, 437, 49 2006, ApJ, 652, 937

Broadhurst, T., et al. 2005a, ApJ, 619, L143 2005b, ApJ, 621, 53

Cacciato, M., Bartelmann, M., Meneghetti, M., \& Moscardini, L. 2006, A\&A, 458,349

Dahle, H., Kaiser, N., Squires, G., \& Broadhurst, T. J. 2001, in ASP Conf. Ser. 237, Gravitational Lensing: Recent Progress and Future Goals, ed. T. G. Brainerd \& C. S. Kochanek (San Francisco: ASP), 311

Diego, J. M., Protopapas, P., Sandvik, H. B., \& Tegmark, M. 2005a, MNRAS, 360,477

Diego, J. M., et al. 2005b, MNRAS, 362, 1247

Duc, P., et al. 2002, A\&A, 382, 60

Dye, S., et al. 2001, MNRAS, 321, 685

Goldberg, D. M., \& Bacon, D. J. 2005, ApJ, 619, 741 (GB05)

Goldberg, D. M., \& Gott, J. R. 2007, Cartographica, in press (astro-ph/ 0608501)

Goldberg, D. M., \& Leonard, A. 2007, ApJ, 660, 1003 (GL07)

Halkola, A., Seitz, S., \& Pannella, M. 2006, MNRAS, 372, 1425 2007, ApJ, 656, 739

Heymans, C., et al. 2006, MNRAS, 368, 1323

Irwin, J., \& Shmakova, M. 2005, NewA Rev., 49, 83 . 2006, ApJ, 645, 17
Kaiser, N., Squires, G., \& Broadhurst, T. 1995, ApJ, 449, 460

King, L. J., Clowe, D. I., \& Schneider, P. 2002a, A\&A, 383, 118

King, L. J., et al. 2002b, A\&A, 385, L5

Kneib, J.-P., et al. 2003, ApJ, 598, 804

Krist, J. 1993, in ASP Conf. Ser. 52, Astronomical Data Analysis Software and Systems II, ed. R. J. Hanisch, R. J. V. Brissenden, \& J. Barnes (San Francisco: ASP), 536

Limousin, M., et al. 2006, ApJ, submitted (astro-ph/0612165) (L06)

Massey, R., \& Refregier, A. 2005, MNRAS, 363, 197

Massey, R., Rowe, B., Refregier, A., Bacon, D. J., \& Berge, J. 2007a, MNRAS, submitted (astro-ph/0609795)

Massey, R., et al. 2007b, Nature, 445, 286

$$
\text { 2007c, MNRAS, 376, } 13
$$

Meurer, G. R., et al. 2003, Proc. SPIE, 4854, 507

Natarajan, P., \& Springel, V. 2004, ApJ, 617, L13

Okura, Y., Umetsu, K., \& Futamase, T. 2007, ApJ, 660, 995

Refregier, A. 2003, MNRAS, 338, 35

Refregier, A., \& Bacon, D. J. 2003, MNRAS, 338, 48

Rix, H., et al. 2004, ApJS, 152, 163

Saha, P., Read, J. I., \& Williams, L. L. R. 2006, ApJ, 652, L5

Seitz, C., \& Schneider, P. 1995, A\&A, 297, 287

Sharon, K., et al. 2005, in IAU Symp. 225, Gravitational Lensing Impact on Cosmology, ed. Y. Mellier \& G. Meylan (Cambridge: Cambridge Univ. Press), 167

Smith, G. P., et al. 2005, MNRAS, 359, 417

Taylor, A. N., \& Dye, S. 1998, NewA Rev., 42, 153

Tyson, J. A., \& Fischer, P. 1995, ApJ, 446, L55

Umetsu, K., Broadhurst, T. J., Takado, M., \& Kong, X. 2005, J. Korean Astron. Soc., 38, 191

Zekser, K., et al. 2006, ApJ, 640, 639 